\title{
Oral Antiplatelet Therapy for Acute and Chronic Management of NSTE ACS: Residual Ischemic Risk and Opportunities for Improvement
}

\author{
Marc Cohen
}

Published online: 5 November 2009

(C) The Author(s) 2009. This article is published with open access at Springerlink.com

\begin{abstract}
Introduction Non-ST-segment elevation acute coronary syndromes (NSTE ACS) are highly prevalent in the United States and globally, and are associated with significant morbidity and mortality.

Discussion The key role of platelet-mediated thrombosis in the pathogenesis of NSTE ACS is confirmed by the proven clinical benefits of antiplatelet agents (aspirin and a $\mathrm{P} 2 \mathrm{Y}_{12}$ adenosine diphosphate [ADP] receptor antagonist) in this setting. Despite the documented advantages and broad use of antiplatelet therapy, the long-term morbidity and mortality rates remain significant, and the bleeding risk remains substantial. Residual risk can be attributed, at least in part, to the fact that thrombosis continues in the presence of current treatments because aspirin and $\mathrm{P} 2 \mathrm{Y}_{12}$ ADP receptor antagonists each block only one of multiple platelet activation pathways, and thus do not impact other platelet activation pathways, such as the one triggered by interaction of thrombin with protease-activated receptor (PAR)-1, thereby exposing patients to continued accumulation of thrombotic events.

Conclusion These considerations suggest that novel therapies with a different mechanism of action, when used in combination with current antiplatelet agents, may provide more comprehensive inhibition of platelet activation and additional reductions in morbidity and mortality, potentially without incremental bleeding risk.
\end{abstract}

M. Cohen $(\bowtie)$

Division of Cardiology, Newark Beth Israel Medical Center,

201 Lyons Avenue at Osborne Terrace,

Newark, NJ 07112, USA

e-mail: marcohen@sbhcs.com
Key words Non-ST-segment elevation acute coronary syndromes · Antiplatelet therapy · PAR-1 · Percutaneous coronary intervention $\cdot$ Bleeding

\section{Introduction}

Non-ST-segment elevation acute coronary syndromes (NSTE ACS), which comprise unstable angina (UA) and non-ST-segment elevation myocardial infarction (NSTEMI), are associated with significant morbidity, mortality and economic burden in the United States. Of the over 1.3 million unique annual hospitalizations for ACS in the United States in 2006, over 800,000 were for myocardial infarction (MI) (approximately two-thirds of these were NSTEMI) and almost 540,000 were for UA [1]. In addition, over 150,000 Americans died from an MI in 2005 [1]. The primary pathophysiological mechanism responsible for clinical manifestations of NSTE ACS involves occlusion of coronary arteries by platelet-rich thrombi, whose generation was triggered in response to injury to vascular endothelium, such as a rupture or erosion of an atherosclerotic plaque [2]. Platelet activation, a key step in platelet thrombus formation, can be initiated by multiple agonists, such as thrombin, thromboxane $\mathrm{A}_{2}$, adenosine diphosphate (ADP) and collagen [3]; the goal of medical therapy is to prevent plateletmediated thrombosis and the resulting acute ischemic events. The key role of platelet-mediated thrombosis in the pathogenesis of NSTE ACS is confirmed by the proven clinical benefits of antiplatelet agents in these patients [4-6]. However, despite the documented clinical efficacy of antiplatelet therapy with aspirin and a $\mathrm{P}_{2} \mathrm{Y}_{12}$ ADP receptor antagonist, the long-term morbidity and mortality associated with NSTE ACS remains significant $[5,6]$, as these agents each block only one of the multiple platelet activation 
pathways leading to thrombotic events; they do not interfere with the pathways stimulated by other platelet activators, including thrombin, the most potent platelet agonist [7, 8]. The stimulatory effect of thrombin on platelet-mediated thrombosis continues even in the presence of aspirin and a P2 $\mathrm{Y}_{12}$ ADP receptor antagonist, thereby potentially leading to thrombotic events. In clinical practice, the residual ischemic risk in patients with NSTE ACS is often further exacerbated by the underuse of antiplatelet agents in spite of their welldocumented benefits [9-11]. Apart from the substantial residual risk for ischemic events, current oral antiplatelet agents are also associated with increased bleeding risk. These considerations underscore the need for more comprehensive prevention of platelet-mediated thrombosis and associated ischemic events, ideally without an incremental bleeding risk.

The aims of this review are to discuss the results and clinical implications of key clinical trials with oral antiplatelet agents in patients with NSTE ACS, as well as to review the emerging evidence regarding the "net clinical benefit" or the balance between bleeding and ischemic events. In addition, this review will also address the current management of NSTE ACS in US clinical practice, based on the findings from the ACTION (NRMI/CRUSADE) and GRACE registries, and the opportunities for improvements in patient care.

\section{Oral antiplatelet therapy: benefits and risks}

Aspirin Aspirin inhibits the activity of the cyclooxygenase (COX)-1 enzyme, thereby limiting the production of thromboxane $\mathrm{A}_{2}$, an important platelet agonist implicated in both pathologic thrombus formation and protective hemostasis [3]. The efficacy of aspirin for treatment of patients presenting with NSTE ACS and for secondary prevention in patients with established atherothrombotic disease has been demonstrated in several clinical trials [1214] and meta-analyses [4]. Aspirin is the foundation of treatment recommendations for both acute and chronic antiplatelet therapy in patients with NSTE ACS $[15,16]$. However, residual morbidity and mortality and bleeding risk remain substantial with aspirin. An observed inverse relationship between aspirin dose and clinical benefit [3] may be attributed to a dose-dependent increase in bleeding risk (mainly gastrointestinal bleeding) [17]. It should be noted that even low aspirin doses $(\leq 100 \mathrm{mg})$ are associated with increased bleeding rates compared with placebo [18]. However, the inverse relationship between aspirin dose and clinical benefit may not rely solely on higher bleeding risk, but also on a trend toward a lower anti-thrombotic benefit at doses higher than $75-100 \mathrm{mg} /$ day [19]. The lack of additional cardiovascular benefit by increasing doses of aspirin has been recently confirmed by results of the CURRENT-OASIS 7 trial (discussed below) [20] and may be attributed to a dose-dependent inhibition of prostacyclin at higher doses of aspirin [21].

$P 2 Y_{12}$ ADP receptor inhibitors These agents, including clopidogrel, prasugrel and ticagrelor, bind to and inhibit the activation of the platelet $\mathrm{P} 2 \mathrm{Y}_{12}$ receptor by its physiological ligand ADP, which is released from activated platelets, and amplifies platelet activation and aggregation [3]. Clopidogrel is a thienopyridine prodrug that is converted into an active compound by cytochrome $\mathrm{P} 450$ isoenzymes in the liver [22]. Addition of clopidogrel to aspirin in patients with NSTE ACS was demonstrated to reduce the risk of adverse ischemic outcomes and to increase bleeding risk versus aspirin alone in the CURE trial (Table 1) [6], as well as in its substudy PCI-CURE in patients with NSTE ACS who have undergone percutaneous coronary intervention (PCI) [23]. On the basis of these findings, the addition of clopidogrel to aspirin has become the standard of care for patients with NSTE ACS, and its use in these patients is recommended by both the American College of Cardiology (ACC)/American Heart Association (AHA) [15] and European guidelines [16]. Furthermore, the benefits of adding clopidogrel to aspirin have also been demonstrated in patients with ST-segment elevation MI [24] and patients undergoing elective PCI [25], as well as in patients with atrial fibrillation who do not wish to or cannot take anticoagulant warfarin [26]. The CHARISMA trial did not demonstrate a significant benefit of clopidogrel plus aspirin vs aspirin alone in the overall population of patients with clinically documented atherothrombotic disease or multiple risk factors [27]; however, a significant reduction in the incidence of ischemic events with the combination of clopidogrel and aspirin was evident in the secondary prevention cohort (ie, in patients with prior MI, stroke or symptomatic peripheral arterial disease) [28]. Most recently, the CURRENT-OASIS 7 trial evaluated the efficacy and safety of high-dose versus standard-dose clopidogrel in patients with ACS [20]. Patients $(n=25,087)$ assigned to high-dose clopidogrel received a 600-mg loading dose and $150 \mathrm{mg}$ once daily for seven days, followed by $75 \mathrm{mg}$ once daily until day 30 . Patients in the standard clopidogrel arm received a 300-mg loading dose, followed by $75 \mathrm{mg}$ once daily until 30 days. After day 30, all patients received clopidogrel $75 \mathrm{mg}$ once-daily. Patients were also randomized to receive low-dose (75 to $100 \mathrm{mg}$ per day) or high-dose (300 to $325 \mathrm{mg}$ per day) aspirin. In the overall study population, there was no significant difference in the primary endpoint (combined rate of death from $\mathrm{CV}$ causes, MI, and stroke) between patients receiving the high-dose and the standard-dose clopidogrel therapy $(4.2 \%$ vs $4.4 \% ; P=0.37)$ [20] In patients who underwent PCI, however, the high-dose 
clopidogrel regimen was associated with a $15 \%$ relative reduction in risk of death from $\mathrm{CV}$ causes, MI, or stroke at 30 days versus the standard-dose regimen $(3.9 \%$ versus $4.5 \% ; P=0.036$ ). No significant differences in efficacy were observed between the high-dose and the standard-dose clopidogrel arms in patients who did not undergo PCI. In patients who underwent PCI, treatment with the high-dose clopidogrel regimen was also associated with significant reductions in stent thrombosis and MI at 30 days No significant differences in TIMI major bleeding, fatal bleeding, intracranial hemorrhage, or CABG-related bleeding were observed between the two clopidogrel treatment arms. As alluded to above, there was no significant difference in the primary outcome in patients allocated high-dose versus low-dose aspirin $(4.2 \%$ vs $4.4 \% ; P=0.47)$ [20]. Of note, rates of TIMI major bleeding were also comparable between the high-dose and low-dose aspirin groups $(0.97 \%$ vs $1.03 \%$; $P=0.71$ ).

Several studies have documented variable responsiveness of platelets to therapy with clopidogrel [29]. Although a standardized definition and methodology for assessment of responsiveness to antiplatelet therapy has not been established, sufficient evidence supports the concept that persistence of enhanced platelet reactivity despite the use of clopidogrel is clinically relevant [30-33]. A correlation between low level of inhibition of ADP-induced platelet aggregation in response to clopidogrel and recurrence of ischemic events has been documented in several studies in patients with ACS and those undergoing PCI [31-33].

Although the mechanisms responsible for the variability and low responsiveness to clopidogrel have not been fully elucidated, recent analyses suggest that genetic polymorphisms of the cytochrome P (CYP) 450 enzymes can significantly modulate individual response to clopidogrel and are important determinants of prognosis [34-36]. A study of patients with acute MI treated with clopidogrel demonstrated that the carriers of the CYP2C19*2 allelic variant (CYP2C19) had a significantly higher rate of ischemic events (death, non-fatal MI, or urgent revascularization) than non-carriers (10.9 events per 100 patient-years vs 2.9 events per 100 patient-years, respectively; adjusted hazard ratio: 5.38, $P<0.0001)$ [34]. Similarly, in a French registry of patients with acute MI treated with clopidogrel, patients with any two loss-of-function CYP2C19 variants had a significantly higher rate of death, non-fatal MI, or stroke versus patients no loss-of-function alleles $(21.5 \%$ vs. 13.3\%; adjusted HR 1.98) [36]. The increased risk was particularly prominent among patients undergoing PCI [36]. Additionally, in the TRITON trial (discussed below), patients treated with clopidogrel who were carriers of at least one reduced-function CYP2C19 allele had a significantly higher rate of cardiovascular events than non-carriers ( $12.1 \%$ vs. $8.0 \%$, respectively; $P=0.01$ ) [35]. Other studies 
have suggested that co-administration of clopidogrel with a proton pump inhibitor, particularly omeprazole, decreases the antiplatelet effects and clinical benefit of clopidogrel [37, 38], but the data supporting this association are mixed [39].

The delayed onset of action and variable inhibition of platelet aggregation with clopidogrel have prompted the search for potentially more effective $\mathrm{P}_{2} \mathrm{Y}_{12}$ ADP receptor antagonists. The first among these newer $\mathrm{P}_{2} \mathrm{Y}_{12}$ ADP receptor antagonists is prasugrel, which is characterized by a faster onset of action and more potent inhibition of ADPinduced platelet aggregation as compared with clopidogrel $[40,41]$. The TRITON-TIMI 38 trial evaluated the efficacy and safety of the combination of prasugrel plus aspirin versus clopidogrel (300-mg loading dose plus 75-mg oncedaily maintenance dose) plus aspirin in patients with ACS scheduled for PCI [5]. In TRITON, treatment with prasugrel plus aspirin was associated with a significantly lower rate of the combined end point of cardiovascular death, non-fatal MI and non-fatal stroke versus treatment with clopidogrel plus aspirin (Table 1) [5]. The net clinical benefit (combined incidence of cardiovascular death, nonfatal MI, non-fatal stroke and non-fatal Thrombolyis in Myocardial Infarction [TIMI] major bleeding not related to coronary artery bypass grafting [CABG] surgery) of prasugrel was particularly pronounced in patients with diabetes (hazard ratio [HR]: $0.74 ; p=0.001$ ) and those presenting with ST-segment elevation MI (HR: $0.81 ; p=$ 0.02 ), largely because these patient groups did not experience an increase in TIMI major bleeding [42, 43]. The incidence of stent thrombosis was also substantially lower in the prasugrel-plus-aspirin arm (Fig. 1) [44]. However, the residual risk for ischemic events with prasugrel plus aspirin in TRITON-TIMI 38 remained substantial $(\approx 10 \%$ at 15 months; Table 1$)$, and the risk of bleeding (TIMI major, life-threatening, fatal, TIMI major and minor, requiring transfusion and $\mathrm{CABG}$ surgeryrelated) in the overall population was significantly higher than with clopidogrel plus aspirin (Table 1) [5]. Furthermore, no net benefit of prasugrel plus aspirin over clopidogrel plus aspirin was observed in patients aged $\geq 75$ years with ACS (HR: $0.99 ; p=0.92$ ) or those weighing $<60 \mathrm{~kg}$ (HR: $1.03 ; p=0.89$ ), and a net clinical harm was apparent in patients with prior stroke or transient ischemic attack (TIA) (HR: $1.54 ; p=0.04$ ) [5], primarily due to higher rates of bleeding with prasugrel plus aspirin than with clopidogrel plus aspirin in these patient groups. These results suggest that the selection of a $\mathrm{P}_{2} \mathrm{Y}_{12}$ ADP receptor antagonist should take into account not only the greater pharmacological potency of prasugrel versus clopidogrel, but also the increased risk for bleeding with prasugrel as compared with clopidogrel. The net harm observed in patients with stroke or TIA suggests that prasugrel should be avoided in this patient group. Likewise, patients at higher bleeding risk, such as the elderly or those with low body weight, may not derive a net benefit from prasugrel. It is important to note that consideration of the use of the 5$\mathrm{mg}$ maintenance dose of prasugrel in patients weighing $<60 \mathrm{~kg}$, as described in the US prescribing information for prasugrel [45], is entirely based on pharmacokinetic modeling and is not supported by any clinical evidence. In selected patients (ie, patients with diabetes or those with STEMI) with ACS undergoing PCI, the combination of prasugrel plus aspirin may provide greater protection against ischemic events than the combination of clopidogrel plus aspirin, although even in these patients the residual risk for ischemic events with prasugrel plus aspirin remained substantial $[42,43]$. On the basis of these results, prasugrel has recently been approved in Europe and in the United States, although the pattern of its use in clinical practice remains to be seen.

In addition to prasugrel, ticagrelor (AZD 6140), a novel non-thienopyridine $\mathrm{P}_{2} \mathrm{Y}_{12}$ ADP receptor antagonist, has also demonstrated a faster onset of action, more potent inhibition of ADP-induced platelet aggregation than clopidogrel, as well as more rapid reversal of inhibition of ADP-induced
Fig. 1 Incidence of stent thrombosis over 15 months in patients receiving prasugrel plus aspirin versus clopidogrel plus aspirin in the TRITON-TIMI 38 trial [44]. Reproduced with permission

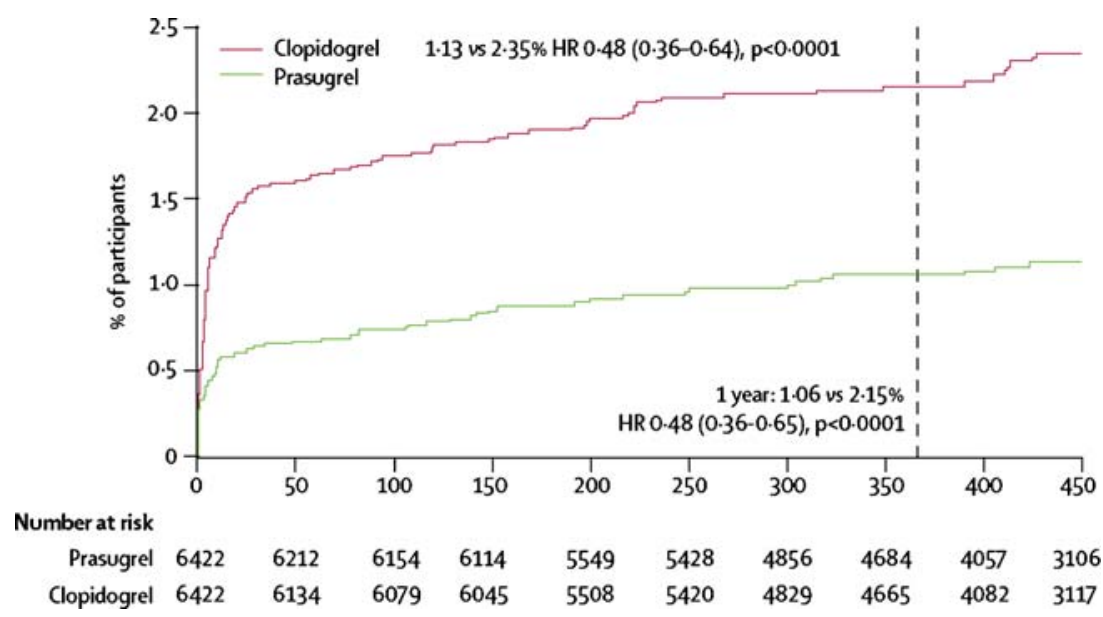


platelet activation and aggregation than clopidogrel [22]. Unlike clopidogrel or prasugrel, ticagrelor is not a prodrug and does not require metabolic conversion into an active metabolite [22]. These properties suggest that ticagrelor may be an attractive alternative to clopidogrel, especially in clinical situations where rapid inhibition of platelet aggregation or its quick reversal may be required. The efficacy and safety of 1-year treatment with ticagrelor (plus aspirin versus clopidogrel plus aspirin was recently reported in patients with ACS in the phase 3 PLATO trial [46] (Table 1). Patients receiving ticagrelor plus aspirin experienced a significant reduction in the incidence of the composite endpoint of first occurrence of $\mathrm{CV}$ death, MI, or stroke versus patients receiving clopidogrel plus aspirin (Table 1). Of note, the rate of all-cause death was $22 \%$ lower with ticagrelor versus clopidogrel $(P<0.001)$. There was no significant difference in rates of major bleeding between the treatment arms (Table 1). Rates of other adverse events were higher with ticagrelor versus clopidogrel: dyspnea $(13.8 \%$ versus $7.8 \%$; $P<0.001)$, syncope ( $1.1 \%$ versus $0.8 \% ; P=0.08)$, ventricular pauses $\geq 3 \mathrm{~s}$ during the first week of treatment $(5.8 \%$ versus $3.6 \% ; P=0.01)$ and increase in serum uric acid and serum creatinine at 1 month and 1 year $(P<0.001$ for each comparison]) [46]. It is important to note that ticagrelor is administered twice daily; in clinical practice, the need for twice-daily dosing may increase the risk for ischemic events in patients who are not fully compliant with the prescribed therapy.

Even in the presence of dual antiplatelet therapy with aspirin and a $\mathrm{P} 2 \mathrm{Y}_{12}$ ADP receptor antagonist (clopidogrel, prasugrel or ticagrelor), the risk for morbidity and mortality remains substantial in patients with NSTE ACS, as well as in those with ST-segment-elevation MI, atrial fibrillation, or a history of prior atherothrombotic disease, and in patients scheduled for PCI. This residual risk can be attributed to the fact that multiple pathways contribute to platelet activation, and aspirin and $\mathrm{P}_{2} \mathrm{Y}_{12}$ ADP receptor antagonists each inhibit only one of these pathways (the thromboxane $A_{2}$ and ADP pathways, respectively). The lack of an inhibitory effect of current therapies on other platelet activation pathways allows continued platelet reactivity in the presence of potent agonists, such as thrombin, thereby increasing the risk for recurrent thrombotic/ischemic events, including death. When used in combination with the current standard-of-care therapies, new agents that target pathways that are not affected by aspirin or $\mathrm{P}_{2} \mathrm{Y}_{12}$ ADP receptor antagonists may provide complementary and more comprehensive inhibition of platelet activation, and thereby contribute to greater inhibition of platelet-mediated thrombosis and incremental reductions in ischemic events. Apart from the residual ischemic risk, dual antiplatelet therapy with aspirin and a $\mathrm{P}_{2} \mathrm{Y}_{12}$ ADP receptor antagonist is also associated with an increased risk of bleeding, because these agents interfere with the thromboxane $\mathrm{A}_{2}$ and ADP platelet activation pathways that are essential for normal hemostasis [3]. These considerations underscore the need for novel antiplatelet agents that provide more comprehensive platelet inhibition without interfering with platelet activation pathways critical for hemostasis, for greater protection against thrombotic events without an incremental bleeding risk.

PAR-1 (thrombin) receptor antagonists PAR-1 is the principal receptor for thrombin on human platelets [47]. Interaction of thrombin, the most potent platelet agonist, with PAR-1 promotes platelet shape change and granule secretion, as well as other processes leading to platelet activation [47]. Preclinical observations indicate that inhibition or genetic inactivation of PAR-1 selectively interferes with platelet activation mediated by thrombin and with platelet deposition into an occlusive thrombus, but not with thrombin-mediated fibrin generation or initial platelet deposition that is important for healing in response to vascular injury [48-50]. These results suggest that platelet activation mediated by PAR-1 may be critical for thrombosis but may not be necessary for hemostasis. PAR-1 inhibitors (or thrombin receptor antagonists [TRAs]) represent a novel class of antiplatelet agents. Currently, two PAR-1 antagonists are in clinical development: E-5555 and SCH 530348. When used in combination with the current standard-of-care antiplatelet therapy (aspirin alone or dual therapy with aspirin and a $\mathrm{P}_{2} \mathrm{Y}_{12}$ ADP receptor antagonist), a PAR-1 inhibitor offers more comprehensive platelet inhibition and potentially an incremental reduction in ischemic events, possibly without a risk of increased bleeding.

E-5555 is an orally active, potent PAR-1 antagonist that has demonstrated antiplatelet effects without increasing bleeding times in preclinical studies [51, 52]. Serebruany et al. evaluated the in vitro effects of E-5555 on platelet aggregation and biomarker expression in blood from healthy volunteers $(n=10)$, patients with documented coronary artery disease (CAD) treated with aspirin $(n=$ 10 ), and patients with documented CAD who were treated with aspirin plus clopidogrel $(n=10)$ [53]. Complete inhibition of TRAP-induced platelet aggregation was observed at all concentrations of E-5555 evaluated (20, 50 , and $100 \mathrm{ng} / \mathrm{ml}$ ), including the lowest dose [53]. Modest inhibition of platelet aggregation induced by ADP and collagen was observed. Four ongoing Phase 2 trials are evaluating the safety and tolerability of daily, oral administration of E-5555 (50 mg, $100 \mathrm{mg}$ or $200 \mathrm{mg}$ ) in patients with CAD or NSTE ACS in the US and Japan [54-57].

SCH 530348 is the first oral PAR-1 inhibitor in phase 3 clinical development [58]. The phase 2 TRA-PCI trial evaluated the safety and efficacy of SCH 530348 (administered as either a 10-, 20-, or 40-mg loading dose followed 
by a maintenance dose of $0.5 \mathrm{mg} \mathrm{qd}, 1 \mathrm{mg}$ qd, or $2.5 \mathrm{mg}$ qd for 59 days) used in combination with standard oral antiplatelet therapy (aspirin and clopidogrel) and an antithrombin agent (heparin or bivalirudin) versus placebo in patients with planned non-urgent PCI [59]. Based on the recommendation of the safety review committee, enrollment of the prespecified 1,600 patients was reduced because of low TIMI major and minor bleeding rates. TRA-PCI randomized a total of 1,030 patients, of which 573 subsequently underwent PCI as planned (primary PCI cohort), whereas the remaining patients either underwent CABG surgery $(n=76)$ or were managed medically $(n=$ 381) [59]. Although the study may be underpowered for safety results, rates of clinically significant bleeding were low overall in TRA-PCI. Administration of SCH 530348 in combination with standard therapy was not associated with increased rates of TIMI major or minor bleeding, the primary endpoint, compared with standard therapy alone in the primary PCI cohort ( $2.8 \%$ vs $3.3 \% ; P=0.77)$ [59]. None of the patients treated with the combination of the highest loading and maintenance doses of SCH 530348 (40-mg loading dose plus 2.5-mg maintenance dose) experienced TIMI major bleeding, although rates of overall bleeding, TIMI minor bleeding and non-TIMI bleeding were numerically higher with SCH 530348. The rate of discontinuations due to non-TIMI bleeding in the primary cohort were similar in the combined SCH 530348 and placebo arms (1.4\% and $1.3 \%$, respectively) [59]. The rate of TIMI major or minor bleeding did not differ significantly between patients allocated SCH 530348 versus placebo who underwent CABG surgery (90\% vs 79\%) [59]. No clear dose-response relationship for post-operative bleeding was evident in surgical patients. More patients who underwent CABG and received SCH 530348 versus placebo needed transfusions; however the proportion of patients who needed $\geq 2$ units of packed red blood cells did not differ between groups. Among patients who were managed medically, TIMI major/minor bleeding was observed in 3 patients allocated SCH 530348 and zero patients allocated placebo [59]. Although not powered to detect differences in efficacy endpoints, a lower incidence of ischemic events, specifically MI, was observed in patients allocated SCH 530348 [59]. In this study, the most rapid onset of action (as measured by inhibition of thrombin receptor agonist peptide [TRAP]-induced platelet aggregation) was provided by the 40-mg loading dose of SCH 530348, whereas the 2.5 -mg once-daily maintenance dose of SCH 530348 subsequently selected for phase 3 trials sustained complete $(>80 \%)$ inhibition of TRAP-induced platelet aggregation over the 60-day treatment period [59]. Importantly, SCH 530348 selectively inhibits platelet aggregation induced by TRAP but does not interfere with the aggregation induced by other platelet agonists, such as arachidonic acid, ADP, or collagen [60]. A separate phase 2 trial in Japanese patients with NSTE ACS scheduled for PCI also demonstrated that the addition of SCH 530348 to the standard-of-care therapy (aspirin plus ticlopidine) did not result in increased bleeding risk and was associated with a significant reduction in periprocedural MI [61]. These results suggest that the use of SCH 530348 in combination with standard antiplatelet therapy (aspirin with or without a P2Y $\mathrm{Y}_{12}$ ADP receptor antagonist) may provide incremental reductions in ischemic events, potentially without an increased risk of bleeding. Two large ongoing phase 3 trials are evaluating the benefits and bleeding risks associated with the addition of SCH 530348 to the standard-of-care therapy (aspirin alone or aspirin plus a $\mathrm{P}_{2} \mathrm{Y}_{12}$ ADP receptor inhibitor) in patients presenting with NSTE ACS $(N \approx 10,000$; ClinicalTrials.gov Identifier: NCT00527943) and in secondary prevention $(N \approx 25,000$ patients; ClinicalTrials.gov Identifier: NCT00526474).

\section{Relationship between bleeding and ischemic events}

An increase in bleeding risk in patients treated with aspirin and $\mathrm{P}_{2} \mathrm{Y}_{12}$ ADP receptor antagonists has been well recognized, and several recent studies suggest that bleeding and blood transfusions may represent independent predictors of short- and long-term mortality in patients with ACS and in those undergoing PCI [62-66]. For example, a 2004 report by Rao and colleagues evaluated the correlation of blood transfusions and morbidity and mortality in over 24,000 patients with ACS, demonstrating that transfusions were a powerful predictor of 30-day mortality (HR: 3.94; 95\% confidence interval [CI] 3.26-4.75; $p<0.001)$ and $30-$ day death/MI (HR: 2.92; 95\% CI 2.55-3.35; $p<0.001$ ) [65]. Similarly, the risk for 30-day and 6-month mortality among over 26,000 patients with NSTE ACS was shown to increase proportionally with greater severity of bleeding [66]. These observations were further confirmed by a pooled analysis of the OASIS registry and the OASIS-2 and CURE trials, which involved over 34,000 patients with NSTE ACS [62]. This study demonstrated that major bleeding was associated with a fivefold increase in the risk of mortality at 30 days (Fig. 2a) [62], and that the risk of death over the 6-month follow-up increased in proportion to the bleeding severity (Fig. 2b) [62]. In addition to the mortality increase, major bleeding was also associated with a significantly increased risk of MI (HR: $4.44 ; 95 \% \mathrm{CI}$ $3.16-6.24 ; p<0.0001$ ) and stroke (HR: 6.46 ; 95\% CI 3.54$11.79 ; p<0.0001)$ within 30 days [62]. In the ACUITY trial, patients with NSTE ACS who experienced major bleeding had significantly higher 30-day incidences of death, composite ischemia and stent thrombosis than patients 

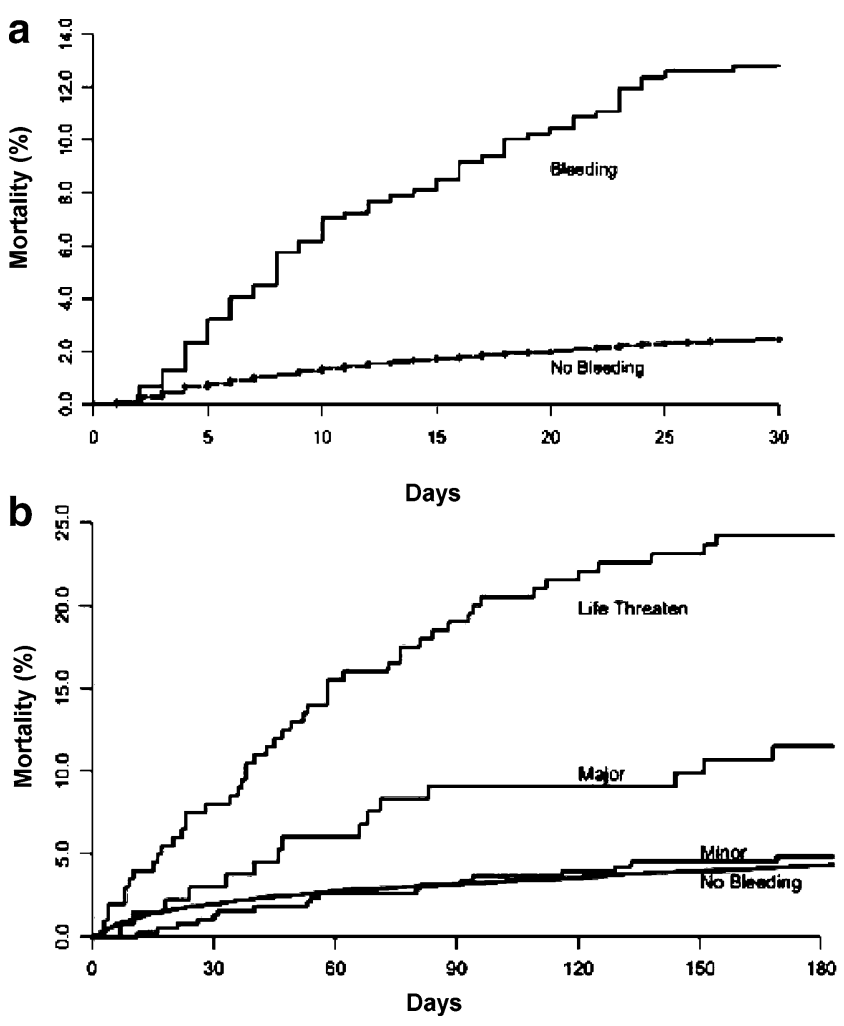

Fig. 2 a Relationship between major bleeding and mortality in a meta-analysis of 34,146 patients with NSTE ACS in the OASIS-1, OASIS-2 and CURE trials during the first 30 days (HR: 5.37; 95\% CI 3.97-7.26; $p<0.0001$ ) [62]. b Relationship between bleeding severity and the risk of death: Kaplan-Meier estimates of mortality among patients who developed no, minor, major (excluding life-threatening) or life-threatening bleeding in the CURE trial ( $p$ for trend $=0.0009$ ) [62]. Reproduced with permission

without major bleeding ( $p<0.0001$ for all 3 outcomes), and major bleeding was the strongest independent predictor of 30-day mortality (HR: 7.55; 95\% CI 4.68-12.18; $p<0.0001$ ) [63]. Additionally, a pooled analysis of 4 ISAR trials in patients undergoing PCI (ISAR-REACT, ISAR-SWEET, ISAR-SMART 2 and ISAR-REACT 2) demonstrated that bleeding within 30 days was an independent predictor of 1year mortality (HR: 2.96; 95\% CI 1.96-4.48; $p<0.001$ ) [64]. Of note, the predictive value of bleeding within 30 days for 1 -year mortality was comparable to that of MI within 30 days (HR: 2.29; 95\% CI 1.52-3.46; $p<0.001$ ) or urgent revascularization within 30 days (HR: 2.49 ; $95 \%$ CI $1.16-5.35$; $p=$ 0.019) [64]. Collectively, these results suggest that bleeding is a significant risk factor for death and ischemic events. The mechanisms linking bleeding and blood transfusions to increased mortality in patients with ACS and patients undergoing PCI are incompletely understood but likely involve multiple factors, including the location and intensity of bleeding, impaired oxygen delivery, as well as discontinuation of antiplatelet therapy [67]. Regardless of the mechanisms and factors that may contribute to the increased risk of mortality associated with bleeding and blood transfusions, it is clear that the approaches designed to minimize the bleeding risk may also result in lower rates of ischemic events and thereby improve overall patient outcomes.

\section{Use of antiplatelet therapy in clinical practice: insights from registries}

Continuous evaluations of management of patients with NSTE ACS in the United States in the CRUSADE registry from 2002 to 2004 have demonstrated significant improvements in use of medications both in the acute setting (antiplatelet agents, anticoagulants, glycoprotein IIb/IIIa receptor inhibitors and beta-blockers) and in the discharge setting (antiplatelet agents, lipid-lowering agents, angiotensin-converting enzyme inhibitors) [11]. However, use of many therapies was suboptimal, and there was a clear need for greater implementation of the ACC/AHA guidelines' recommendations [11]. CRUSADE has also documented significantly lower use of evidence-based therapies in the elderly, women, minority populations, and patients without private insurance [68-70]. More recently, the ACTION registry reported that clopidogrel was used in only $60 \%$ of patients with NSTEMI in the acute setting and in 74\% of patients with NSTEMI at discharge during 2008 [71]. Differences in clopidogrel utilization have also been noted based on management strategy [70, 71]. The latest available data from ACTION for the year 2008 document that clopidogrel was used among patients with NSTEMI at the time of hospital discharge in $97 \%$ of those who underwent PCI, but in only $55 \%$ of those who were medically managed, and in only $28 \%$ of those who underwent $\mathrm{CABG}$, even though they were admitted to the hospital with an ACS [71]. Importantly, lack of early clopidogrel use was

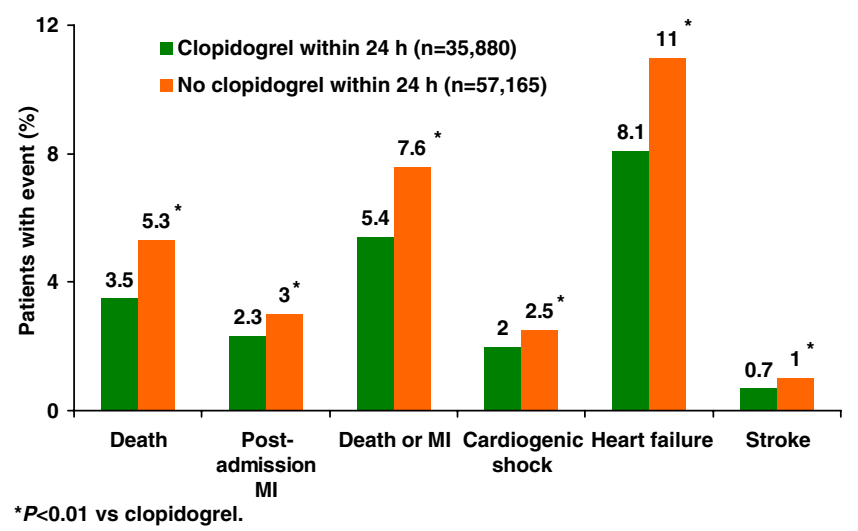

Fig. 3 In-hospital outcomes in 93,045 patients with NSTEMI not undergoing PCI by pattern of clopidogrel use in CRUSADE [9]. $p<$ 0.01 for all comparisons 
associated with significantly higher in-hospital mortality and other adverse outcomes compared with early initiation of clopidogrel in CRUSADE (Fig. 3) [9].

The global GRACE registry of over 27,000 patients with NSTE ACS in 14 countries has reported significant reductions in clinical events and increased utilization of medical therapies and PCI between 1999-2000 and 2005 [10]. These improvements included significant decreases in the rates of in-hospital death $(2.9 \%$ in $1999-2000$ versus $2.2 \%$ in $2005 ; p=0.02$ ) and 6 -month mortality $(4.9 \%$ in $1999-2000$ versus $3.3 \%$ in 2005 ; $p=$ $0.04)$ [10]. The rates of in-hospital congestive heart failure or pulmonary edema, MI, cardiogenic shock and 6-month stroke were also significantly lower (all $p<0.05$ ) in 2005 than in 1999-2000, while the in-hospital rates of stroke and 6-month rates of MI did not differ significantly [10]. These reductions in clinical events may be attributed, at least in part, to considerable increases in adherence to guidelines-based use of thienopyridines and lipid-lowering agents, as recommended by the US and European guidelines [10].

Registry data have documented considerable advances in patient care and improved clinical outcomes. Nevertheless, in-hospital mortality rates remain substantial in the United States $(4.1 \%$ in patients with NSTEMI in ACTION) and globally $(2.2 \%$ in patients with NSTE ACS in GRACE) $[10,71]$. Despite the ACC/AHA guidelines' recommendations [15], clopidogrel initiation is often postponed until after diagnostic catheterization and withheld at the time of discharge in patients who are managed medically and those who undergo surgical revascularization. Thus, there is considerable room for greater adherence to the guidelines' recommendations for the use of oral antiplatelet therapy among patients with NSTE ACS in the acute and particularly in the chronic setting, which can lead to improved patient outcomes. At the same time, bleeding and blood transfusions, which are associated with increased mortality risk, remain a frequent complication in patients with NSTE ACS. For example, major bleeding and red blood cell transfusion occurred during 2008 in the ACTION registry in $12 \%$ and $15 \%$ of patients with NSTEMI, respectively [71], while GRACE reported major bleeding in $4.7 \%$ of patients with NSTEMI and $2.3 \%$ of patients with UA [72]. Unadjusted in-hospital death rates in GRACE in patients with NSTEMI with major bleeding versus no bleeding were $15.3 \%$ versus $5.3 \%(p<0.001)$ [72]. These considerations suggest that the clinical benefits of greater use of current oral antiplatelet therapies recommended by the guidelines may be partially offset by the increased risk for bleeding or blood transfusions, and thereby underscore a critical need for novel therapies that reduce the risk of ischemic events without exacerbating the risk of bleeding complications.

\section{Conclusions}

Clinical trials and registry data have documented clear beneficial effects of current oral antiplatelet agents (aspirin and $\mathrm{P}_{2} \mathrm{Y}_{12}$ ADP receptor inhibitors) on ischemic outcomes in patients with NSTE ACS. However, despite the proven clinical efficacy of these agents, residual morbidity and mortality remain substantial even in patients receiving dual antiplatelet therapy. This residual ischemic risk may be explained by the fact that these agents interfere only with the thromboxane $\mathrm{A}_{2}$ and ADP platelet activation pathways and do not block platelet-mediated thrombosis stimulated by other platelet activators (such as thrombin), allowing the accumulation of ischemic events. In addition to a high residual risk for ischemic events, aspirin and $\mathrm{P}_{2} \mathrm{Y}_{12}$ ADP receptor antagonists are associated with increased bleeding risk, which can be attributed to the inhibitory effect of these agents on pathways essential for hemostasis. Novel therapeutic approaches such as the PAR-1 inhibitors, targeting platelet activation pathways not affected by current antiplatelet agents, represent an attractive strategy to reduce the residual ischemic risk in patients with NSTE ACS, possibly without exacerbating the risk of bleeding. In addition to novel therapeutic approaches, improvements in clinical outcomes in standard practice can also be achieved through the greater use of currently available antiplatelet agents with proven efficacy, such as aspirin and clopidogrel, particularly in patients who are managed without PCI. The benefits of increased use of currently recommended oral antiplatelet agents, though, should be balanced against the increased risk for bleeding complications. The availability of oral antiplatelet agents with a more favorable benefit-to-risk profile than the currently available therapies would represent a major advance in the treatment of patients with NSTE ACS.

Acknowledgements Editorial assistance was provided by Gina Fusaro, PhD, and Joshua Barbach, MA, of Health Science Communications. This assistance was funded by Schering-Plough. The author did not receive any financial compensation for this work and is fully responsible for the content of the manuscript.

Open Access This article is distributed under the terms of the Creative Commons Attribution Noncommercial License which permits any noncommercial use, distribution, and reproduction in any medium, provided the original author(s) and source are credited.

\section{References}

1. Lloyd-Jones D, Adams R, Carnethon M, et al. Heart disease and stroke statistics - 2009 update: a report from the American Heart Association Statistics Committee and Stroke Statistics Subcommittee. Circulation. 2009;119:e21-e181.

2. Libby P, Theroux P. Pathophysiology of coronary artery disease. Circulation. 2005;111:3481-88. 
3. Davi G, Patrono C. Platelet activation and atherothrombosis. N Engl J Med. 2007;357:2482-94.

4. Baigent C, Blackwell L, Collins R, et al. Aspirin in the primary and secondary prevention of vascular disease: collaborative metaanalysis of individual participant data from randomised trials. Lancet. 2009;373:1849-60.

5. Wiviott SD, Braunwald $\mathrm{E}, \mathrm{McCabe} \mathrm{CH}$, et al. Prasugrel versus clopidogrel in patients with acute coronary syndromes. N Engl J Med. 2007;357:2001-15.

6. Yusuf S, Zhao F, Mehta SR, Chrolavicius S, Tognoni G, Fox KK. Effects of clopidogrel in addition to aspirin in patients with acute coronary syndromes without ST-segment elevation. N Engl J Med. 2001;345:494-502.

7. Brummel KE, Paradis SG, Butenas S, Mann KG. Thrombin functions during tissue factor-induced blood coagulation. Blood. 2002;100:148-52.

8. Mann KG. Thrombin formation. Chest. 2003;124:4S-10S.

9. Alexander D, Ou FS, Roe MT, et al. Use of and inhospital outcomes after early clopidogrel therapy in patients not undergoing an early invasive strategy for treatment of non-ST-segment elevation myocardial infarction: results from Can Rapid risk stratification of Unstable angina patients Suppress ADverse outcomes with Early implementation of the American College of Cardiology/American Heart Association guidelines (CRUSADE). Am Heart J. 2008;156:606-12.

10. Fox KA, Steg PG, Eagle KA, et al. Decline in rates of death and heart failure in acute coronary syndromes, 1999-2006. JAMA. 2007;297:1892-900.

11. Mehta RH, Roe MT, Chen AY, et al. Recent trends in the care of patients with non-ST-segment elevation acute coronary syndromes: insights from the CRUSADE initiative. Arch Intern Med. 2006;166:2027-34.

12. Cairns JA, Gent M, Singer J, et al. Aspirin, sulfinpyrazone, or both in unstable angina. Results of a Canadian multicenter trial. N Engl J Med. 1985;313:1369-75.

13. Lewis HD Jr, Davis JW, Archibald DG, et al. Protective effects of aspirin against acute myocardial infarction and death in men with unstable angina. Results of a Veterans Administration Cooperative Study. N Engl J Med. 1983;309:396-403.

14. The RISC Group. Risk of myocardial infarction and death during treatment with low dose aspirin and intravenous heparin in men with unstable coronary artery disease. Lancet. 1990;336:827-30.

15. Anderson JL, Adams CD, Antman EM, et al. ACC/AHA 2007 guidelines for the management of patients with unstable angina/ non-ST-Elevation myocardial infarction: a report of the American College of Cardiology/American Heart Association Task Force on Practice Guidelines (Writing Committee to Revise the 2002 Guidelines for the Management of Patients With Unstable Angina/Non-ST-Elevation Myocardial Infarction) developed in collaboration with the American College of Emergency Physicians, the Society for Cardiovascular Angiography and Interventions, and the Society of Thoracic Surgeons endorsed by the American Association of Cardiovascular and Pulmonary Rehabilitation and the Society for Academic Emergency Medicine. J Am Coll Cardiol. 2007;50:e1-e157.

16. Bassand JP, Hamm CW, Ardissino D, et al. Guidelines for the diagnosis and treatment of non-ST-segment elevation acute coronary syndromes. Eur Heart J. 2007;28:1598-660.

17. Campbell CL, Smyth S, Montalescot G, Steinhubl SR. Aspirin dose for the prevention of cardiovascular disease: a systematic review. JAMA. 2007;297:2018-24.

18. Peters RJ, Mehta SR, Fox KA, et al. Effects of aspirin dose when used alone or in combination with clopidogrel in patients with acute coronary syndromes: observations from the Clopidogrel in Unstable angina to prevent Recurrent Events (CURE) study. Circulation. 2003;108:1682-87.
19. Antithrombotic Trialists' Collaboration. Collaborative metaanalysis of randomised trials of antiplatelet therapy for prevention of death, myocardial infarction, and stroke in high risk patients. BMJ. 2002;324:71-86.

20. Mehta SR. CURRENT OASIS 7: a $2 \times 2$ factorial randomized trial of optimal clopidogrel and aspirin dosing in patients with ACS undergoing an early invasive strategy with intent for PCI. Presented at: European Society of Cardiology (ESC) 2009 Congress. August 29-September 2, 2009. Barcelona, Spain.

21. FitzGerald GA, Oates JA, Hawiger J, et al. Endogenous biosynthesis of prostacyclin and thromboxane and platelet function during chronic administration of aspirin in man. J Clin Invest. 1983;71:676-88

22. Raju NC, Eikelboom JW, Hirsh J. Platelet ADP-receptor antagonists for cardiovascular disease: past, present and future. Nat Clin Pract Cardiovasc Med. 2008;5:766-80.

23. Mehta SR, Yusuf S, Peters RJ, et al. Effects of pretreatment with clopidogrel and aspirin followed by long-term therapy in patients undergoing percutaneous coronary intervention: the PCI-CURE study. Lancet. 2001;358:527-33.

24. Sabatine MS, Cannon CP, Gibson CM, et al. Addition of clopidogrel to aspirin and fibrinolytic therapy for myocardial infarction with ST-segment elevation. N Engl J Med. 2005;352:1179-89.

25. Steinhubl SR, Berger PB, Mann JT 3rd, et al. Early and sustained dual oral antiplatelet therapy following percutaneous coronary intervention: a randomized controlled trial. JAMA. 2002;288: 2411-20

26. Connolly S, Pogue J, Hart R, et al. Effect of clopidogrel added to aspirin in patients with atrial fibrillation. N Engl J Med. 2009;360: $1-13$.

27. Bhatt DL, Fox KA, Hacke W, et al. Clopidogrel and aspirin versus aspirin alone for the prevention of atherothrombotic events. N Engl J Med. 2006;354:1706-17.

28. Bhatt DL, Flather MD, Hacke W, et al. Patients with prior myocardial infarction, stroke, or symptomatic peripheral arterial disease in the CHARISMA trial. J Am Coll Cardiol. 2007;49:1982-88.

29. Serebruany VL, Steinhubl SR, Berger PB, Malinin AI, Bhatt DL, Topol EJ. Variability in platelet responsiveness to clopidogrel among 544 individuals. J Am Coll Cardiol. 2005;45:246-51.

30. Angiolillo DJ, Fernandez-Ortiz A, Bernardo E, et al. Variability in individual responsiveness to clopidogrel: clinical implications, management, and future perspectives. J Am Coll Cardiol. 2007;49:1505-16.

31. Cuisset T, Frere C, Quilici J, et al. Benefit of a 600-mg loading dose of clopidogrel on platelet reactivity and clinical outcomes in patients with non-ST-segment elevation acute coronary syndrome undergoing coronary stenting. J Am Coll Cardiol. 2006;48:1339-45.

32. Hochholzer W, Trenk D, Bestehorn HP, et al. Impact of the degree of peri-interventional platelet inhibition after loading with clopidogrel on early clinical outcome of elective coronary stent placement. J Am Coll Cardiol. 2006;48:1742-50.

33. Matetzky S, Shenkman B, Guetta V, et al. Clopidogrel resistance is associated with increased risk of recurrent atherothrombotic events in patients with acute myocardial infarction. Circulation. 2004;109:3171-75.

34. Collet JP, Hulot JS, Pena A, et al. Cytochrome P450 2C19 polymorphism in young patients treated with clopidogrel after myocardial infarction: a cohort study. Lancet. 2009;373:309-17.

35. Mega JL, Close SL, Wiviott SD, et al. Cytochrome p-450 polymorphisms and response to clopidogrel. $\mathrm{N}$ Engl $\mathrm{J}$ Med. 2009;360:354-62.

36. Simon T, Verstuyft C, Mary-Krause M, et al. Genetic determinants of response to clopidogrel and cardiovascular events. N Engl J Med. 2009;360:363-75. 
37. Gilard M, Arnaud B, Cornily JC, et al. Influence of omeprazole on the antiplatelet action of clopidogrel associated with aspirin: the randomized, double-blind OCLA (Omeprazole CLopidogrel Aspirin) study. J Am Coll Cardiol. 2008;51:256-60.

38. Ho PM, Maddox TM, Wang L, et al. Risk of adverse outcomes associated with concomitant use of clopidogrel and proton pump inhibitors following acute coronary syndrome. JAMA. 2009;301: 937-44.

39. O'Donoghue ML, Braunwald E, Antman EM, et al. Pharmacodynamic effect and clinical efficacy of clopidogrel and prasugrel with or without a proton-pump inhibitor: an analysis of two randomised trials. Lancet. 2009;374:989-97.

40. Jernberg T, Payne CD, Winters KJ, et al. Prasugrel achieves greater inhibition of platelet aggregation and a lower rate of nonresponders compared with clopidogrel in aspirin-treated patients with stable coronary artery disease. Eur Heart J. 2006;27:116673.

41. Wallentin L, Varenhorst C, James S, et al. Prasugrel achieves greater and faster P2Y12receptor-mediated platelet inhibition than clopidogrel due to more efficient generation of its active metabolite in aspirin-treated patients with coronary artery disease. Eur Heart J. 2008;29:21-30.

42. Montalescot G, Wiviott SD, Braunwald E, et al. Prasugrel compared with clopidogrel in patients undergoing percutaneous coronary intervention for ST-elevation myocardial infarction (TRITON-TIMI 38): double-blind, randomised controlled trial. Lancet. 2009;373:723-31.

43. Wiviott SD, Braunwald E, Angiolillo DJ, et al. Greater clinical benefit of more intensive oral antiplatelet therapy with prasugrel in patients with diabetes mellitus in the trial to assess improvement in therapeutic outcomes by optimizing platelet inhibition with prasugrel-Thrombolysis in Myocardial Infarction 38. Circulation. 2008;118:1626-36.

44. Wiviott SD, Braunwald $\mathrm{E}, \mathrm{McCabe} \mathrm{CH}$, et al. Intensive oral antiplatelet therapy for reduction of ischaemic events including stent thrombosis in patients with acute coronary syndromes treated with percutaneous coronary intervention and stenting in the TRITON-TIMI 38 trial: a subanalysis of a randomised trial. Lancet. 2008;371:1353-63.

45. Sankyo D, Lilly E et al. Effient (prasugrel) Prescribing Information. 2010.

46. Wallentin L, Becker RC, Budaj A, et al. Ticagrelor versus clopidogrel in patients with acute coronary syndromes. N Engl J Med. 2009;361:1045-57.

47. Coughlin SR. Protease-activated receptors in hemostasis, thrombosis and vascular biology. J Thromb Haemost. 2005;3:1800-14.

48. Derian CK, Damiano BP, Addo MF, et al. Blockade of the thrombin receptor protease-activated receptor-1 with a smallmolecule antagonist prevents thrombus formation and vascular occlusion in nonhuman primates. J Pharmacol Exp Ther. 2003;304:855-61.

49. Kato Y, Kita Y, Hirasawa-Taniyama Y, et al. Inhibition of arterial thrombosis by a protease-activated receptor 1 antagonist, FR171113, in the guinea pig. Eur J Pharmacol. 2003;473:163-9.

50. Vandendries ER, Hamilton JR, Coughlin SR, Furie B, Furie BC. Par4 is required for platelet thrombus propagation but not fibrin generation in a mouse model of thrombosis. Proc Natl Acad Sci U S A. 2007;104:288-92.

51. Kai Y, Hirano K, Maeda Y, Nishimura J, Sasaki T, Kanaide H. Prevention of the hypercontractile response to thrombin by proteinase-activated receptor-1 antagonist in subarachnoid hemorrhage. Stroke. 2007;38:3259-65.
52. Kogushi M, Kobayashi H, Matsuoka T. Antithrombotic and bleeding time effects of E5555, an orally active proteaseactivated receptor-1 antagonist, in guinea pigs. Circulation. 2003;108:280.

53. Serebruany VL, Kogushi M, Dastros-Pitei D, Flather M, Bhatt DL. The in-vitro effects of E5555, a protease-activated receptor (PAR)-1 antagonist, on platelet biomarkers in healthy volunteers and patients with coronary artery disease. Thromb Haemost. 2009;102:111-9.

54. Safety and Tolerability of E5555 and Its Effects on Markers of Intravascular Inflammation in Subjects With Coronary Artery Disease. NCT00312052. At: http://www.clinicaltrials.gov/ct2/ show/NCT00312052?term $=\mathrm{e}-5555 \&$ rank $=3$. Accessed July 17, 2009.

55. Safety and Tolerability of E5555 and Its Effects on Markers of Intravascular Inflammation in Subjects With Acute Coronary Syndrome. NCT00548587. At: http://www.clinicaltrials.gov/ct2/ show/NCT00548587?term $=$ e-5555\&rank=4. Accessed July 17, 2009.

56. A Double-Blind Study of E5555 in Japanese Patients With Acute Coronary Syndrome. NCT00619164. At: http://www.clinicaltrials. gov/ct2/show/NCT00619164?term $=\mathrm{e}-5555 \&$ rank $=1$. Accessed July 17, 2009.

57. A Double-Blind Study of E5555 in Japanese Subjects With Coronary Artery Disease. NCT00540670. At: http://www.clinical trials. gov $/ \mathrm{ct} 2 /$ show $/$ NCT00540670? term $=e-5555 \&$ rank $=2$. Accessed July 17, 2009.

58. Chackalamannil S, Wang Y, Greenlee WJ, et al. Discovery of a novel, orally active himbacine-based thrombin receptor antagonist (SCH 530348) with potent antiplatelet activity. J Med Chem. 2008;51:3061-4.

59. Becker RC, Moliterno DJ, Jennings LK, et al. Safety and tolerability of SCH 530348 in patients undergoing non-urgent percutaneous coronary intervention: a randomised, double-blind, placebo-controlled phase II study. Lancet. 2009;373: 919-28.

60. Jennings LK, Earhart A, Becker RC, et al. Thrombin receptor antagonist (TRA;SCH530348) is a selective, potent inhibitor of PAR1 activity with predictable pharmacokinetics. [abstract]. Circulation. 2007;116:11-674.

61. Goto S, Yamaguchi T, Ikeda Y, Yamaguchi H, Shimizu K, Jensen P. Phase II trial of the novel antiplatelet agent, SCH 530348, in Japanese patients with non-ST segment elevation acute coronary syndromes (NSTE ACS). [abstract]. Eur Heart J. 2008;29:829.

62. Eikelboom JW, Mehta SR, Anand SS, Xie C, Fox KA, Yusuf S. Adverse impact of bleeding on prognosis in patients with acute coronary syndromes. Circulation. 2006;114:774-82.

63. Manoukian SV, Feit F, Mehran R, et al. Impact of major bleeding on 30-day mortality and clinical outcomes in patients with acute coronary syndromes: an analysis from the ACUITY Trial. J Am Coll Cardiol. 2007;49:1362-68.

64. Ndrepepa G, Berger PB, Mehilli J, et al. Periprocedural bleeding and 1-year outcome after percutaneous coronary interventions: appropriateness of including bleeding as a component of a quadruple end point. J Am Coll Cardiol. 2008;51:690-97.

65. Rao SV, Jollis JG, Harrington RA, et al. Relationship of blood transfusion and clinical outcomes in patients with acute coronary syndromes. JAMA. 2004;292:1555-62.

66. Rao SV, O'Grady K, Pieper KS, et al. Impact of bleeding severity on clinical outcomes among patients with acute coronary syndromes. Am J Cardiol. 2005;96:1200-6.

67. Doyle BJ, Rihal CS, Gastineau DA, Holmes DR Jr. Bleeding, blood transfusion, and increased mortality after percutaneous 
coronary intervention: implications for contemporary practice. $\mathrm{J}$ Am Coll Cardiol. 2009;53:2019-27.

68. Peterson ED, Pollack CV Jr, Roe MT, et al. Early use of glycoprotein IIb/IIIa inhibitors in non-ST-elevation acute myocardial infarction: observations from the National Registry of Myocardial Infarction 4. J Am Coll Cardiol. 2003;42:45-53.

69. Sonel AF, Good CB, Mulgund J, et al. Racial variations in treatment and outcomes of black and white patients with high-risk non-ST-elevation acute coronary syndromes: insights from CRUSADE (Can Rapid Risk Stratification of Unstable Angina Patients
Suppress Adverse Outcomes With Early Implementation of the ACC/AHA Guidelines?). Circulation. 2005;111:1225-32.

70. Tricoci P, Roe MT, Mulgund J, et al. Clopidogrel to treat patients with non-ST-segment elevation acute coronary syndromes after hospital discharge. Arch Intern Med. 2006;166:806-11.

71. ACTION Registry. ACTION Registry-GWTG Results Q1 2008 through Q4 2008. 2009. Accessed June 5, 2009.

72. Moscucci M, Fox KA, Cannon CP, et al. Predictors of major bleeding in acute coronary syndromes: the Global Registry of Acute Coronary Events (GRACE). Eur Heart J. 2003;24:1815-23. 